\title{
Predictive scores for hepatocellular carcinoma: a race with no winners?
}

\author{
Raffaella Tortora, Marco Guarracino, Giovan Giuseppe Di Costanzo \\ Liver Unit, Cardarelli Hospital, Naples, Italy \\ Correspondence to: Giovan Giuseppe Di Costanzo. Liver Unit, Cardarelli Hospital, Via A Cardarelli 9, 80131, Naples, Italy. \\ Email: dicostanzogg@gmail.com. \\ Comment on: Wang Z, Fan Q, Wang M, et al. Comparison between Child-Pugh Score and albumin-bilirubin grade in patients treated with the \\ combination therapy of transarterial chemoembolization and sorafenib for hepatocellular carcinoma. Ann Transl Med 2020;8:537.
}

Submitted May 15, 2020. Accepted for publication Jun 28, 2020.

doi: 10.21037/atm-20-3960

View this article at: http://dx.doi.org/10.21037/atm-20-3960

Cancer is a major public health problem with an estimated 18.1 million new cases and 9.6 million cancer deaths during 2018 , worldwide (1). It is a heterogeneous group of diseases thus prediction models have been constructed to help clinicians in identifying subgroups of patients with different survival and therapy response. Identification of prognostic factors is crucial for planning treatment and stratification of patients enrolled in studies. In several cancers, discovery of biomarkers forecasting drug response has changed the treatment landscape. This revolution only partially involved hepatocellular carcinoma (HCC) for which identification of reliable predictive models and biomarkers is still controversial. Such gap is confirmed by the high number of staging and prognostic models proposed during the last 35 years (Table 1). HCC is a unique neoplasm developing mainly in cirrhosis and prognosis prediction is a complex task because it may be influenced by tumor burden, liver disfunction and complications of portal hypertension cirrhosis-related (17). Furthermore, such prediction should be dynamically evaluated being influenced by treatment and changing prevalence of cancer progression and liver failure. With this scenario, it is likely that a single score or system does not fit all clinical conditions.

To increase the complexity, it should be considered that scores are not universally applicable being influenced by characteristics of population used to identify prognostic variables. Okuda et al. formulated the first score combining tumor burden and liver function (2). However, the definition of tumor burden (less or more than $50 \%$ of liver volume) is too rough to be applied in clinical practice today.
The widespread use of imaging identifies a rising number of small HCC and Okuda system is useless to classify these cases. To ameliorate the accuracy, the Cancer of the Liver Italian Program (CLIP) score evaluates also the variables cancer multifocality, AFP and portal thrombosis (3). But again, tumor morphology is roughly defined and CLIP is unsuitable to classify small tumors that may receive curative treatments. The French scoring system (GETCH) is few validated and barely used (4). Chinese University Prognostic Index (CUPI) introduced TNM stage to characterize tumor morphology. However, it performs better in advanced cases being developed from a patient cohort mainly with advanced HCC (6). The Japan Integrated Staging (JIS) and Stage Liver damage DEs- $\gamma$-carboxy-prothrombin (SLIDE) scores include TNM staging by Liver Cancer Study Group of Japan $(7,8)$. JIS is simple and easily calculable whereas SLIDE incorporates indocyanine green and des- $\gamma$-carboxyprothrombin tests not widely available. Tokyo score performs better in patients receiving curative treatments being developed from patients treated by percutaneous ablation (9). To reduce possible imaging-related bias, BALAD score and its modification were constructed using only serum biomarkers to characterize tumor burden and aggressiveness $(10,14)$. Taipei score combines Child-Pugh score with total tumor volume, but external validation is lacking (11). The Model to Estimate Survival in Ambulatory HCC Patients (MESIAH) includes only objective parameters and the resulting score is continuous allowing an accurate stratification of patients independent by performed treatment or etiology $(12,18)$. The model to 
Table 1 Clinical scores and systems for predicting prognosis in HCC patients

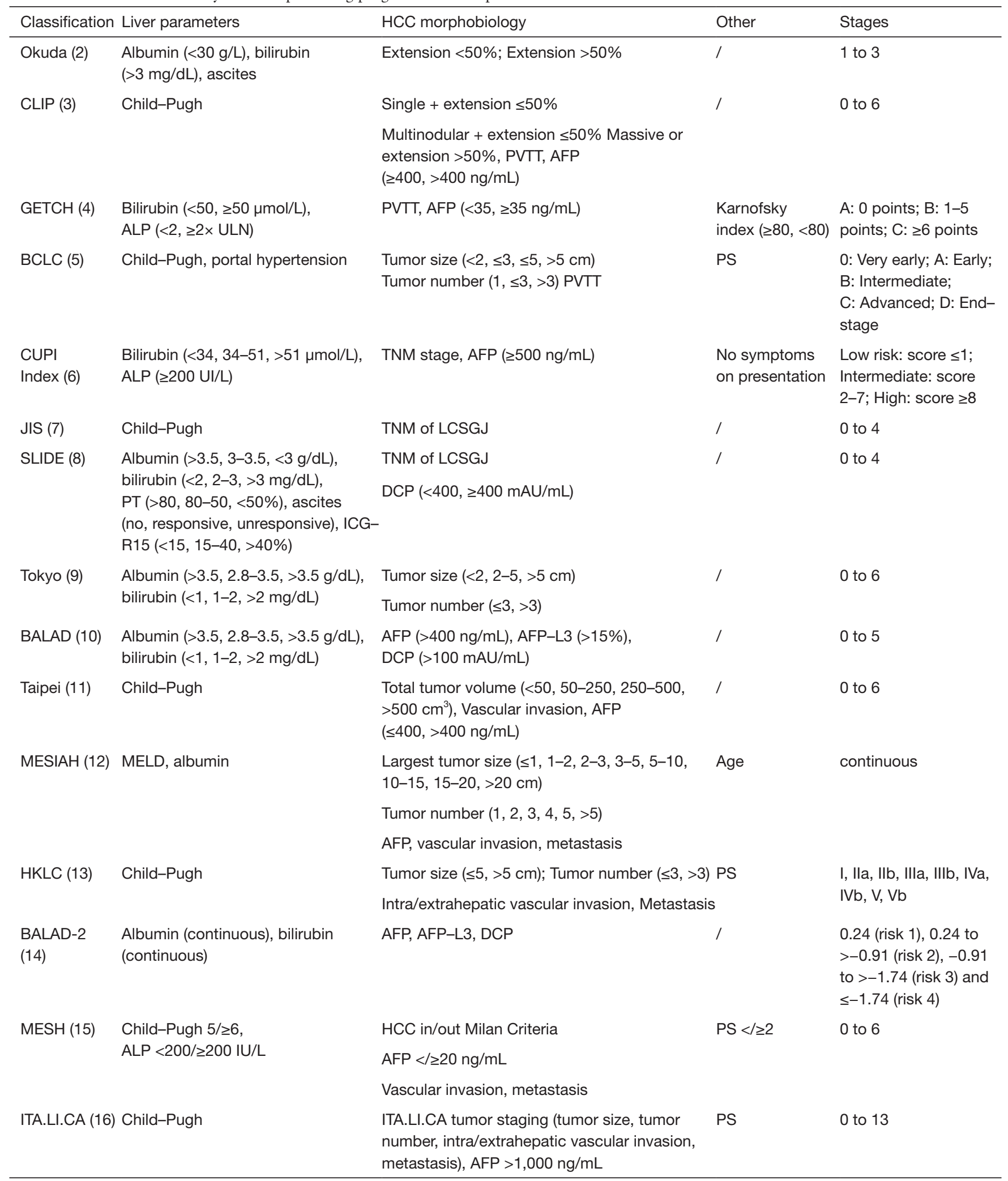

HCC, hepatocellular carcinoma; DCP, Des- $\gamma$-carboxy prothrombin; PVTT, portal vein tumor thrombosis; ICG-R15, indocyanine green 15-minute clearance retention rate; ALP, alkaline phosphatase; LCSGJ, Liver Cancer Study Group of Japan. 
Table 2 Clinical scores for predicting prognosis of HCC patients treated with TACE

\begin{tabular}{|c|c|c|c|c|c|}
\hline Classification & Liver parameters & HCC morphobiology & Treatment & Other & Stages/scores \\
\hline HAP (23) & $\begin{array}{l}\text { Albumin }<3.6 \mathrm{~g} / \mathrm{dL} \\
\text { bilirubin }>0.9 \mathrm{mg} / \mathrm{dL}\end{array}$ & Tumor size $>7 \mathrm{~cm}$, AFP $>400 \mathrm{ng} / \mathrm{mL}$ & TAE/cTACE & / & $A, B, C, D$ \\
\hline ABCR (25) & $\begin{array}{l}\text { Increase Child-Pugh } \\
\text { score } \geq 2\end{array}$ & $\begin{array}{l}\mathrm{BCLC}(\mathrm{A}, \mathrm{B}, \mathrm{C}) \text {; AFP (>200 ng/mL); } \\
\text { Radiologic response }\end{array}$ & $\begin{array}{l}\text { cTACE; } \\
\text { Only retreatment }\end{array}$ & / & -3 to +6 \\
\hline mHAP-II (26) & $\begin{array}{l}\text { Albumin }<3.6 \mathrm{~g} / \mathrm{dL} \\
\text { bilirubin }>0.9 \mathrm{mg} / \mathrm{dL}\end{array}$ & $\begin{array}{l}\text { Tumor size }>7 \mathrm{~cm} \text {, Tumor number } \geq 2 \text {, } \\
\text { AFP }>400 \mathrm{ng} / \mathrm{mL}\end{array}$ & CTACE & / & $A, B, C, D$ \\
\hline SNACOR (28) & Child-Pugh (A, B) & $\begin{array}{l}\text { Tumor size }(<5, \geq 5 \mathrm{~cm}) \text {, Tumor number } \\
(<4, \geq 4) \text {, AFP }(<400, \geq 400 \mathrm{ng} / \mathrm{mL}) \text {, } \\
\text { Radiologic response }(C R+P R, S D+P D)\end{array}$ & $\begin{array}{l}\text { cTACE; } \\
\text { Only retreatment }\end{array}$ & I & $0-2,3-6,7-10$ \\
\hline Six-and-twelve (29) & / & Tumor size + number & cTACE & / & $\leq 6,7-12,>12$ \\
\hline
\end{tabular}

HCC, hepatocellular carcinoma; TACE, transarterial chemoembolization.

estimate survival for HCC patients (MESH) incorporates commonly-used clinical variables that are dichotomized for easy calculation with a good discriminative capacity (15). The Barcelona Clinic Liver Cancer (BCLC) is the most widely used system and has been endorsed by EASL and AASLD as the standard staging system (2). Differently from other systems, it was constructed from results of studies not from variables derived by statistical analysis. BCLC system has some drawbacks as lack of discriminatory ability among $\mathrm{B}$ and $\mathrm{C}$ stages that include a heterogeneous population with varying degree of tumor burden, liver damage and survival probability. BCLC system gained popularity because it is simple and guide treatment allocation, being each stage connected to a treatment recommendation. However, this algorythm is too rigid to be applied as it is in daily clinical practice: it does not contemplate the use of combined treatments and in several cases stage migration strategy should be used (19). The Hong Kong Liver Cancer (HKLC) classification seems partially to overcome some problems of BCLC system allowing a better stratification of $B$ and $C$ stage patients in subgroups with different prognosis (13). It was constructed analysing patients predominantly $\mathrm{HBV}$ infected and recently validated in European patients with prevalent alcoholic and $\mathrm{HCV}$ etiology (20). As BCLC system, HKLC links any stage to a treatment recommendation, but some of suggested application as surgery and TACE for BCLC B and C stage patients needs to be validated before clinical application. Recently, a new system including a tumor staging and a prognostic score has been constructed, the Italian Liver Cancer (ITA.LI.CA) prognostic score (16). It seems to have better discriminative ability than BCLC and HKLC allowing a more accurate stratification of patients useful to select the best therapeutic strategy in the single case.

Unfortunately, at diagnosis only $30-40 \%$ of patients have early-stage disease and receive curative treatments. The majority are affected by unresectable or multifocal HCC and the most widely used treatment is transarterial chemoembolization (TACE) (21). This group of patients is extremely heterogeneous with varied tumor burden and survival. The high number of TACE treatments raised interest in formulating scores to improve selection of patients suitable for TACE and to avert over-treatment or procedural-related toxicity (Table 2). They may be divided in two groups: scores to guide the decision for first TACE and scores for TACE re-treatment. Among baseline scores, Hepatoma Arterial-embolisation Prognostic (HAP) was the first score constructed for predicting post-TACE 
outcomes (23). It was modified with the introduction of variable multifocality (mHAP-II) (26) and with evaluation of variables as continuous parameters (mHAP-III) to increase the individual prognostic estimation (27). A webbased calculator for easy prediction of prognosis according to mHAP-III (http://www.livercancer.eu/mhap3.html) was constructed. Recently, a simple score based on tumor diameter and number, six-and-twelve score, was calculated in a large cohort of Asian patients with preserved liver function, but it lacks of validation (29). To identify patients unsuitable for the first TACE, an easy to calculate score, the selection for transarterial chemoembolisation treatment (STATE) score, was developed (24).

Among scores to predict retreatment, ART and ABCR scores differentiate two groups with different survival and risk of major adverse events after the second TACE $(22,25)$. The sequential use of STATE and ART-score (STARTstrategy) was proposed to select patients who benefit from TACE. However, ART and ABCR predictive value was not confirmed in a large European cohort (30). The SNACOR score in addition to basal parameters included HCC response at imaging (28), but its predictive value was not confirmed in a European cohort (31).

In this issue of Annals of Translational Medicine, Wang et al. compared the prognostic value of ALBI model and Child-Pugh score in the specific setting of Child-Pugh A patients who received combined treatment with TACE and sorafenib (32). This therapy is used in clinical practice also if previous randomized clinical trials gave inconsistent results (33-36). The recently published TACTICS trial showed that TACE plus sorafenib as compared to TACE alone increased progression free survival (37). Assessment of liver function before administration of TACE and sorafenib is crucial because patients are exposed to hepatic toxic effects of both therapies. Usually clinicians grade liver function using Child-Pugh classification. It was formulated to evaluate outcomes in cirrhotic patients who receive surgery for portal hypertension but hides several drawbacks. For example, ascites and encephalopathy grade is subjective and cutoff points of biochemical tests are arbitrarily defined. Furthermore, Child-Pugh score works better in patients with liver failure who are excluded from treatment with TACE and sorafenib.

Albumin-Bilirubin (ALBI) grade model is a new tool to evaluate liver function that was formulated from analysis of large international databases (38). It has the advantage over Child-Pugh score of being derived by statistical analysis and not influenced by subjectivity. A nomogram for easy calculation was constructed and resulting linear predictor was categorized into three grades related to distinct prognostic groups. ALBI model performs better in ChildPugh A patients as shown in studies of curative treatments for HCC $(39,40)$. Therefore, we fully agree with the choice of using ALBI model in patients who receive sorafenib plus TACE combination.

In conclusion, there is none prognostic index universally applicable to HCC patients, but it should be selected on the basis of the characteristics of the population and of the planned treatment.

\section{Acknowledgments}

Funding: None.

\section{Footnote}

Provenance and Peer Review: This article was commissioned by the editorial office, Annals of Translational Medicine. The article did not undergo external peer review.

Conflicts of Interest: The authors have completed the ICMJE uniform disclosure form (available at http://dx.doi. org/10.21037/atm-20-3960). The authors have no conflicts of interest to declare.

Ethical Statement: The authors are accountable for all aspects of the work in ensuring that questions related to the accuracy or integrity of any part of the work are appropriately investigated and resolved.

Open Access Statement: This is an Open Access article distributed in accordance with the Creative Commons Attribution-NonCommercial-NoDerivs 4.0 International License (CC BY-NC-ND 4.0), which permits the noncommercial replication and distribution of the article with the strict proviso that no changes or edits are made and the original work is properly cited (including links to both the formal publication through the relevant DOI and the license). See: https://creativecommons.org/licenses/by-nc-nd/4.0/.

\section{References}

1. Bray F, Ferlay J, Soerjomataram I, et al. Global cancer statistics 2018: GLOBOCAN estimates of incidence and mortality worldwide for 36 cancers in 185 countries. CA: A Cancer Journal for Clinicians 2018;68:394-424. 
2. Okuda K, Ohtsuki T, Obata H, et al. Natural history of hepatocellular carcinoma and prognosis in relation to treatment. Study of 850 patients. Cancer 1985;56:918-28.

3. The Cancer of the Liver Italian Program (CLIP) Investigators. A new prognostic system for hepatocellular carcinoma: a retrospective study of 435 patients. Hepatology 1998;28:751-5.

4. Chevret S, Trinchet JC, Mathieu D, et al. A new prognostic classification for predicting survival in patients with hepatocellular carcinoma. Groupe d'Etude et de Traitement du Carcinome Hepatocellulaire. J Hepatol 1999;31:133-41.

5. Llovet JM, Brú C, Bruix J. Prognosis of hepatocellular carcinoma: the BCLC staging classification. Semin Liver Dis $1999 ; 19: 329-38$.

6. Leung TW, Tang AM, Zee B, et al. Construction of the Chinese University Prognostic Index for hepatocellular carcinoma and comparison with the TNM staging system, the Okuda staging system, and the Cancer of the Liver Italian Program staging system: a study based on 926 patients. Cancer 2002;94:1760-9.

7. Kudo M, Chung H, Osaki Y. Prognostic staging system for hepatocellular carcinoma (CLIP score): its value and limitations, and a proposal for a new staging system, the Japan Integrated Staging Score (JIS score). J Gastroenterol 2003;38:207-15.

8. Omagari K, Honda S, Kadokawa Y, et al. Preliminary analysis of a newly proposed prognostic scoring system (SLiDe score) for hepatocellular carcinoma. J Gastroenterol Hepatol 2004;19:805-11.

9. Tateishi R, Yoshida H, Shiina S, et al. Proposal of a new prognostic model for hepatocellular carcinoma: an analysis of 403 patients. Gut 2005;54:419-25.

10. Toyoda H, Kumada T, Osaki Y, et al. Staging hepatocellular carcinoma by a novel scoring system (BALAD score) based on serum markers. Clin Gastroenterol Hepatol 2006;4:1528-36.

11. Hsu CY, Huang YH, Hsia CY, et al. A new prognostic model for hepatocellular carcinoma based on total tumor volume: the Taipei Integrated Scoring System. J Hepatol 2010;53:108-17.

12. Yang JD, Kim WR, Park KW, et al. Model to estimate survival in ambulatory patients with hepatocellular carcinoma. Hepatology 2012;56:614-21.

13. Yau T, Tang VY, Yao TJ, et al. Development of Hong Kong Liver Cancer staging system with treatment stratification for patients with hepatocellular carcinoma. Gastroenterology 2014;146:1691-700.e3.
14. Fox R, Berhane S, Teng M, et al. Biomarker-based prognosis in hepatocellular carcinoma: validation and extension of the BALAD model. Br J Cancer 2014;110:2090-8.

15. Liu PH, Hsu CY, Hsia CY, et al. Proposal and validation of a new model to estimate survival for hepatocellular carcinoma patients. Eur J Cancer 2016;63:25-33.

16. Farinati F, Vitale A, Spolverato G, et al. Development and Validation of a New Prognostic System for Patients with Hepatocellular Carcinoma. PLoS Med 2016;13:e1002006.

17. Bruix J, Sherman M. Management of hepatocellular carcinoma. Hepatology 2005;42:1208-36.

18. Choi WM, Yu SJ, Ahn H, et al. A model to estimate survival in ambulatory patients with hepatocellular carcinoma: Can it predict the natural course of hepatocellular carcinoma? Dig Liver Dis 2017;49:1273-9.

19. European Association for the Study of the Liver. EASL clinical practice guidelines: management of hepatocellular carcinoma. J Hepatol 2018;69:182-236.

20. Heinrich S, Sprinzl M, Schmidtmann I, et al. Validation of prognostic accuracy of MESH, HKLC, and BCLC classifications in a large German cohort of hepatocellular carcinoma patients. United European Gastroenterol J 2020;8:444-52.

21. Park JW, Chen M, Colombo M, et al. Global patterns of hepatocellular carcinoma management from diagnosis to death: the BRIDGE Study. Liver Int 2015;35:2155-66.

22. Sieghart $W$, Hucke F, Pinter $M$, et al The ART of decision making: retreatment with transarterial chemoembolization in patients with hepatocellular carcinoma. Hepatology 2013;57:2261-73.

23. Kadalayil L, Benini R, Pallan L, et al. A simple prognostic scoring system for patients receiving transarterial embolisation for hepatocellular cancer. Ann Oncol 2013;24:2565-70.

24. Hucke F, Pinter M, Graziadei I, et al. How to STATE suitability and START transarterial chemoembolization in patients with intermediate stage hepatocellular carcinoma. J Hepatol 2014;61:1287-96.

25. Adhoute $X$, Penaranda G, Naude S, et al. Retreatment with TACE: the ABCR SCORE, an aid to the decisionmaking process. J Hepatol 2015;62:855-62.

26. Park Y, Kim SU, Kim BK et al. Addition of tumor multiplicity improves the prognostic performance of the hepatoma arterial embolization prognostic score. Liver Int 2016;36:100-7.

27. Cappelli A, Cucchetti A, Cabibbo G, et al. Refining prognosis after trans-arterial chemo-embolization for 
hepatocellular carcinoma. Liver Int 2016;36:729-36.

28. Kim BK, Shim JH, Kim SU, et al. Risk prediction for patients with hepatocellular carcinoma undergoing chemoembolization: development of a prediction model. Liver Int 2016;36:92-9.

29. Wang Q, Xia D, Bai W, et al. Development of a prognostic score for recommended TACE candidates with hepatocellular carcinoma: a multicentre observational study. J Hepatol 2019;70:893-903.

30. Kloeckner R, Pitton MB, Dueber C, et al. Validation of clinical scoring systems ART and ABCR after transarterial chemoembolization of hepatocellular carcinoma. J Vasc Interv Radiol 2017;28:94-102.

31. Mähringer-Kunz A, Weinmann A, Schmidtmann I, et al. Validation of the SNACOR clinical scoring system after transarterial chemoembolisation in patients with hepatocellular carcinoma. BMC Cancer 2018;18:489.

32. Wang Z, Fan Q, Wang M, et al. Comparison between Child-Pugh Score and albumin bilirubin grade in patients treated with the combination therapy of transarterial chemoembolization and sorafenib for hepatocellular carcinoma. Ann Transl Med 2020;8:537.

33. Park JW, Koh YH, Kim HB, et al. Phase II study of concurrent transarterial chemoembolization and sorafenib in patients with unresectable hepatocellular carcinoma. $\mathrm{J}$ Hepatol 2012;56:1336-42.

34. Kudo M, Imanaka K, Chida N, et al. Phase III study of sorafenib after transarterial chemoembolisation in Japanese and Korean patients with unresectable hepatocellular carcinoma. Eur J Cancer 2011;47:2117-27.

35. Lencioni R, Llovet JM, Han G, et al. Sorafenib or placebo plus TACE with doxorubicin-eluting beads for intermediate stage HCC: the space trial. J Hepatol 2016;64:1090-8.

36. Meyer T, Fox R, Ma YT, et al. Sorafenib in combination with transarterial chemoembolisation in patients with unresectable hepatocellular carcinoma (TACE 2): a randomised placebo-controlled, double-blind, phase 3 trial. Lancet Gastroenterol Hepatol 2017;2:565-75.

37. Kudo M, Ueshima K, Ikeda M, et al. Randomised, multicentre prospective trial of transarterial chemoembolisation (TACE) plus sorafenib as compared with TACE alone in patients with hepatocellular carcinoma: TACTICS trial. Gut 2020;69:1492-501.

38. Johnson PJ, Berhane S, Kagebayashi C, et al. Assessment of liver function in patients with hepatocellular carcinoma: a new evidence-based approach-the ALBI grade. J Clin Oncol 2015;33:550-8.

39. Wang YY, Zhong JH, Su ZY, et al. Albumin-bilirubin versus Child-Pugh score as a predictor of outcome after liver resection for hepatocellular carcinoma. Br J Surg 2016;103:725-34.

40. Kao WY, Su CW, Chiou YY, et al. Hepatocellular Carcinoma: Nomograms based on the Albumin-Bilirubin grade to assess the outcomes of radiofrequency ablation. Radiology 2017;285:670-80.
Cite this article as: Tortora R, Guarracino M, Di Costanzo GG. Predictive scores for hepatocellular carcinoma: a race with no winners? Ann Transl Med 2020;8(19):1211. doi: 10.21037/ atm-20-3960 